\title{
Analytical study of holographic p-wave superfluid models in Gauss-Bonnet gravity
}

\author{
Chuyu Lai ${ }^{1, a}$, Tangmei He ${ }^{1}$, Qiyuan Pan ${ }^{2,3, b}$, Jiliang Jing ${ }^{2,3, c}$ \\ ${ }^{1}$ Center for Astrophysics, School of Physics and Electronic Engineering, Guangzhou University, Guangzhou 510006, China \\ ${ }^{2}$ Key Laboratory of Low Dimensional Quantum Structures and Quantum Control of Ministry of Education, Department of Physics, Synergetic \\ Innovation Center for Quantum Effects and Applications, Hunan Normal University, Changsha 410081, Hunan, China \\ ${ }^{3}$ Center for Gravitation and Cosmology, College of Physical Science and Technology, Yangzhou University, Yangzhou 225009, China
}

Received: 1 November 2019 / Accepted: 22 February 2020 / Published online: 17 March 2020

(C) The Author(s) 2020

\begin{abstract}
In Gauss-Bonnet gravity, we analytically investigate the p-wave superfluid models in five dimensional AdS soliton and AdS black hole in order to explore the influences of the higher curvature correction on the holographic superfluid phase transition. We observe that the analytical findings are in good agreement with the numerical computations. Our results show that the critical chemical potential of the system increases with the increase of the Gauss-Bonnet parameter in AdS soliton background, while the critical temperature decreases as the Gauss-Bonnet factor grows if the phase transition of the system is of the second order in AdS black hole background, both of which indicate that the higher curvature correction hinders the formation of the condensation of the vector operator. Moreover, the critical exponent of the system takes the mean-field value $1 / 2$, which is independent of the Gauss-Bonnet parameter and the spatial component of the gauge field.
\end{abstract}

\section{Introduction}

The emergence of the anti-de Sitter/conformal field theory (AdS/CFT) correspondence [1-4], which connects the gravity on asymptotically anti-de Sitter spacetime to the conformal field theory on the $(d-1)$-dimensional boundary of this spacetime, provides a dual description for a strongly interacting system from the perspective of classical gravity. Through this correspondence, there is a great progress in comprehending the dynamics of strongly coupled gauge theories. One of the remarkable applications of the AdS/CFT duality is the study of high-temperature superconductor in

\footnotetext{
a e-mail: laichuyu@gzhu.edu.cn (corresponding author)

be-mail: panqiyuan@126.com

c e-mail: jljing@hunnu.edu.cn
}

condensed matter physics [5]. The core mechanism that is responsible for the holographic superconductor is the spontaneous breaking of $U(1)$ symmetry at the horizon of bulk black hole, which implies the superconducting phase transition in the dual CFTs. In the past decade, a variety of holographic superconductors have attracted considerable attention and been explored. The holographic superconductor in the background of the bulk AdS black hole can be used to model phase transition from normal state to superconducting state, the critical temperature and critical phenomena were studied in Refs. [6-11]. By considering the background of AdS soliton, the holographic models describing the insulator/superconductor transition at zero temperature have been constructed, and the investigations on the properties of these superconductors were carried out in Refs. [12-17]. The studies were also generalized to holographic models of superfluid, in which the spatial component of the $U(1)$ gauge field on the boundary is turned on and it corresponds to the current along the same spatial direction, for reviews, see Refs. [18-23].

Most of the holographic models are built and investigated based on the Einstein gravity. In order to gain an insight into the influences of the higher curvature correction on the superconductor or superfluid phase transition, we will extend the investigation to the higher dimensional spacetime in GaussBonnet (GB) gravity [24]

$$
\begin{aligned}
S= & \frac{1}{16 \pi G} \int d^{d} x \sqrt{-g}\left[R+\frac{(d-1)(d-2)}{L^{2}}\right. \\
& \left.+\alpha\left(R_{\mu \nu \gamma \delta} R^{\mu \nu \gamma \delta}-4 R_{\mu \nu} R^{\mu \nu}+R^{2}\right)\right],
\end{aligned}
$$

where $\alpha$ is the GB coupling constant with dimension $(\text { length })^{2}$. The motivation for this is due to the contradiction between the Mermin-Wagner theorem that forbids spontaneously symmetry breaking at finite temperature in spatial 
dimensions $d \leq 2$ on the gauge theory side and the indeed observation that the superconducting phase transition exists in the four-dimensional AdS black hole backgrounds [5,25]. It is possible that higher curvature correction should suppress condensation based on the suppression of the large fluctuations in the large $N$ limit. According to the AdS/CFT dictionary, the higher derivative curvature terms correspond to the corrections of large $N$ expansion of boundary CFTs in the strong coupling limit. In this direction, lots of work studying various holographic superconductors in EinsteinGauss-Bonnet gravity have been performed and the effects of the GB correction on the phase transition have been discovered [26-35]. Recently, Nam studied $d$-dimensional holographic superconductors in the probe limit in the framework of Einstein-Gauss-Bonnet gravity as well as exponential nonlinear electrodynamics, and found that the GB correction makes the formation of condensation harder, and the superconducting energy gap becomes larger when increasing GB parameter [36]. Ref. [37] numerically investigated the holographic p-wave superconductors with GB curvature correction and nonlinear electrodynamics, and further disclosed the effect of the GB parameter on the behavior of conductivity. In the literature [38], we studied the holographic p-wave superfluid model in the background of soliton solution to Einstein-Maxwell theory. The chemical potential, as a parameter of the soliton solution, has a critical value where a second-order transition will be triggered and the AdS soliton will reach the superconductor (or superfluid) phase. Also from the analysis of the critical phenomenon, it was found that the spatial component of the $U(1)$ gauge field will not bring up the first-order transition. It is of interest to further consider the holographic p-wave superfluid model in AdS soliton including higher order curvature correction. The variation method for the Sturm-Liouville (S-L) eigenvalue problem, which was firstly proposed by Siopsis et al. $[39,40]$ to analytically study the critical phenomenon in holographic superconductor model, is generalized to the studies of various holographic models and proved to be an effective approach, see Refs. [10,14,15,29,33,41-44]. The analytical results obtained from the $\mathrm{S}-\mathrm{L}$ method are in great agreement with the numerical findings. We will employ the analytical S-L method to reveal some details of the holographic $\mathrm{p}$-wave superfluid model in GB AdS soliton and disclose several general properties.

On the other hand, a holographic superfluid solution was constructed based on the AdS black hole background by Herzog et al. [19], and they found that the second-order superfluid phase transition can change to the first order when the velocity of the superfluid component increases relative to the normal component. By coupling a Maxwell-complex vector field into the AdS black hole, Wu et al. investigated the holographic p-wave superfluid and detected that the lager mass of the vector field leads to the larger translating superfluid velocity from second order to first order, and the Cave of Winds only exists in the case of five-dimensioned spacetime [23]. The holographic p-wave superfluid in AdS black hole background in GB gravity has been studied numerically by Liu et al. recently [45]. It revealed that the GB parameter will make it easier for the appearance of translating point from the second-order transition to the first-order one or for the emergence of the Cave of Winds. In particular, for the sufficiently large mass of the vector field, the phase transition of the system is always of the second order. In order to back up this significant feature by analytical calculation, we use the $\mathrm{S}-\mathrm{L}$ method to analytically reproduce the properties of the holographic p-wave superfluid model in GB AdS black hole with the large mass of the vector field fixed where the superfluid phase transition is of the second order. In our paper, we will work in the probe limit where the gravitational backreaction of the vector fields on the background geometry is neglected.

This paper is organized as follows. In the next section, we introduce holographic model of superfluid in GB AdS soliton background. We focus on the influences of the higher curvature correction on the critical chemical potential and the critical phenomena of the system via the S-L method. We also study the behavior of the spatial component of the gauge field near the critical point. In Sect. 3, we extend the analytical study to the p-wave superfluid model in GB AdS black hole with the large mass of the vector field. We calculate the critical temperature as well as the condensation of the vector operator to back up the numerical findings and analyze the behavior of the spatial component of the gauge field. In Sect. 4, we summarise the general properties of the holographic p-wave superfluid model in GB gravity with our main results.

\section{Holographic p-wave superfluid model in Gauss-Bonnet AdS soliton background}

Since we will discuss the holographic superfluid dual to the five-dimensional GB AdS soliton configuration [46], in the probe limit, we begin with the soliton solution to the action (1) for $d=5$ in the form

$d s^{2}=-r^{2} d t^{2}+\frac{d r^{2}}{f(r)}+f(r) d \varphi^{2}+r^{2}\left(d x^{2}+d y^{2}\right)$,

where the metric function

$f(r)=\frac{r^{2}}{2 \alpha}\left[1-\sqrt{1-\frac{4 \alpha}{L^{2}}\left(1-\frac{r_{s}^{4}}{r^{4}}\right)}\right]$,

with the tip $r_{s}$ is a conical singularity in this solution, $\alpha$ is the Gauss-Bonnet coupling constant and $L$ is the AdS radius. In the asymptotic region $(r \rightarrow \infty)$, the function $f(r)$ behaves as 
$f(r) \sim \frac{r^{2}}{2 \alpha}\left(1-\sqrt{1-\frac{4 \alpha}{L^{2}}}\right)$,

so we can define the effective asymptotic AdS scale by

$L_{e f f}^{2}=\frac{2 \alpha}{1-\sqrt{1-\frac{4 \alpha}{L^{2}}}}$,

where the upper bound of the GB parameter $\alpha=L^{2} / 4$ is the so-called Chern-Simons limit [47]. Given the constraints of the causality via the holographic correspondence $[48,49]$, we will take the range $-7 L^{2} / 36 \leq \alpha \leq 9 L^{2} / 100$ for the GB coupling constant in the following calculation. It should be noted that the solution (2) goes back to the Schwarzschild AdS soliton in the Einstein limit, i.e., $\alpha \rightarrow 0$. In order to remove the singularity at the tip, we impose a period $\beta=\pi L^{2} / r_{s}$ for the coordinate $\varphi$ [46], which is the same as that of Schwarzschild AdS soliton [50]. This period remains unchanged as the Gauss-Bonnet parameter varies, which means that we can fix the period of the spatial coordinate $\varphi$ to discuss the influence of the high order correction on the holographic model. For simplicity, we will scale $L=1$ in the following.

In the GB AdS soliton background, considering a Maxwell field and a charged complex vector field coupled, we build the holographic p-wave model of superfluidity via the action

$$
\begin{aligned}
S= & \int d^{5} x \sqrt{-g}\left(-\frac{1}{4} F_{\mu \nu} F^{\mu \nu}-\frac{1}{2} \rho_{\mu \nu}^{\dagger} \rho^{\mu \nu}\right. \\
& \left.-m^{2} \rho_{\mu}^{\dagger} \rho^{\mu}+i q \gamma \rho_{\mu} \rho_{\nu}^{\dagger} F^{\mu \nu}\right),
\end{aligned}
$$

where the tensor $\rho_{\mu \nu}=D_{\mu} \rho_{\nu}-D_{\nu} \rho_{\mu}$ with the covariant derivative $D_{\mu}=\nabla_{\mu}-i q A_{\mu}, q$ and $m$ are the charge and mass of the vector field $\rho_{\mu}$, respectively. In this work the parameter $\gamma$, which is the magnetic moment of the vector field, will not be considered. In order to take the possibility of DC supercurrent into account, we adopt the ansatz of the guage field $A_{\mu}$ as

$A_{\mu} d x^{\mu}=A_{t}(r) d t+A_{\varphi}(r) d \varphi$,

where both a time component $A_{t}$ and a spatial component $A_{\varphi}$ of the vector potential have been introduced. For the vector field $\rho_{\mu}$, we assume it to be real and take the following ansatz

$\rho_{\mu} d x^{\mu}=\rho_{x}(r) d x$.

Therefore we can get a set of equations of motion for the holographic p-wave superfluid model

$$
\begin{aligned}
& \rho_{x}^{\prime \prime}+\left(\frac{1}{r}+\frac{f^{\prime}}{f}\right) \rho_{x}^{\prime}-\frac{1}{f}\left(m^{2}+\frac{q^{2} A_{\varphi}^{2}}{f}-\frac{q^{2} A_{t}^{2}}{r^{2}}\right) \rho_{x}=0, \\
& A_{t}^{\prime \prime}+\left(\frac{1}{r}+\frac{f^{\prime}}{f}\right) A_{t}^{\prime}-\frac{2 q^{2} \rho_{x}^{2}}{r^{2} f} A_{t}=0,
\end{aligned}
$$

$$
A_{\varphi}^{\prime \prime}+\frac{3}{r} A_{\varphi}^{\prime}-\frac{2 q^{2} \rho_{x}^{2}}{r^{2} f} A_{\varphi}=0
$$

where the prime denotes the derivative with respect to $r$. The effective mass of the vector field obtained from the equation of motion for $\rho_{x}$ reads

$m_{e f f}^{2}=m^{2}+\frac{q^{2} A_{\varphi}^{2}}{f}-\frac{q^{2} A_{t}^{2}}{r^{2}}$.

Evidently, the expression of the effective mass implies that the p-wave superfluid phase transition depends on $m^{2}, A_{t}$ and $A_{\varphi}$. The specific results can be deduced from the following computation.

The boundary conditions at the tip of AdS soliton and the asymptotic AdS boundary have to be imposed to solve the nonlinear equations (9). At the tip $r \rightarrow r_{s}$, the solutions behave as

$$
\begin{aligned}
& \rho_{x}(r)=\tilde{\rho}_{x 0}+\tilde{\rho}_{x 1}\left(r-r_{s}\right)+\tilde{\rho}_{x 2}\left(r-r_{s}\right)^{2}+\cdots, \\
& A_{t}(r)=\tilde{A}_{t 0}+\tilde{A}_{t 1}\left(r-r_{s}\right)+\tilde{A}_{t 2}\left(r-r_{s}\right)^{2}+\cdots, \\
& A_{\varphi}(r)=\tilde{A}_{\varphi 1}\left(r-r_{s}\right)+\tilde{A}_{\varphi 2}\left(r-r_{s}\right)^{2}+\cdots .
\end{aligned}
$$

At the asymptotic AdS boundary $r \rightarrow \infty$, we have the constraints

$$
\begin{aligned}
& \rho_{x}(r)=\frac{\rho_{x+}}{r^{\Delta+}}+\frac{\rho_{x-}}{r^{\Delta-}}, \\
& A_{t}(r)=\mu-\frac{\rho}{r^{2}}, \\
& A_{\varphi}(r)=S_{\varphi}-\frac{J_{\varphi}}{r^{2}},
\end{aligned}
$$

where the characteristic exponent $\Delta_{ \pm}=1 \pm \sqrt{1+m^{2} L_{e f f}^{2}}$, $\mu$ and $S_{\varphi}$ are interpreted as the chemical potential and the superfluid velocity, while $\rho$ and $J_{\varphi}$ stand for the charge density and the current in the dual field theory, respectively. The quantities $\rho_{x-}$ and $\rho_{x+}$, according to the AdS/CFT dictionary, correspond to the source and the vacuum expectation value of the vector operator $\left\langle O_{x}\right\rangle$, respectively. Since we expect that the condensate appears spontaneously, we concentrate on the case of $\rho_{x-}=0$ and $\left\langle O_{x}\right\rangle=\rho_{x+}$ in our discussion.

In this system, there are several useful scaling symmetries

$$
\begin{aligned}
& r \rightarrow \lambda r, \quad(t, \varphi, x, y) \rightarrow \frac{1}{\lambda}(t, \varphi, x, y), \quad q \rightarrow q, \\
& \quad\left(\rho_{x}, A_{t}, A_{\varphi}\right) \rightarrow \lambda\left(\rho_{x}, A_{t}, A_{\varphi}\right),
\end{aligned}
$$

which lead to the transformation of the relevant quantities

$$
\begin{gathered}
\left(\mu, S_{\varphi}\right) \rightarrow \lambda\left(\mu, S_{\varphi}\right), \quad\left(\rho, J_{\varphi}\right) \rightarrow \lambda^{3}\left(\rho, J_{\varphi}\right), \\
\rho_{x+} \rightarrow \lambda^{\Delta_{+}+1} \rho_{x+},
\end{gathered}
$$

where $\lambda$ is a real positive number. Using the scaling symmetries (13) we can assume $q=1$ and $r_{s}=1$ without losing the generality in the following numerical calculation. 


\subsection{Critical chemical potentials}

It was found that $[27,51,52]$, for the AdS soliton, the chemical potential $\mu$ of the system has a critical value $\mu_{c}$, above which the solution becomes unstable to develop a hair. It implies the appearance of the condensation of the vector field $\rho_{x}$ in gravity side and the dual field theory reaches a superconductor (or superfluid) phase. For lower chemical potential $\mu<\mu_{c}$, the vector condensate vanishes, and the dual theory is in an insulator phase because in this model the normal phase is described by an AdS soliton where the system exhibits a mass gap. The critical chemical potential is a turning point of the insulator/superconductor phase transitions. To proceed further, we would like to rewrite the equations of motion (9) in the new coordinates $z=r_{s} / r$, so they yield

$$
\begin{aligned}
\rho_{x}^{\prime \prime}+ & \left(\frac{1}{z}+\frac{f^{\prime}}{f}\right) \rho_{x}^{\prime} \\
+ & {\left[\frac{1}{z^{2} f}\left(\frac{q A_{t}}{r_{s}}\right)^{2}-\frac{1}{z^{4} f^{2}}\left(\frac{q A_{\varphi}}{r_{s}}\right)^{2}-\frac{m^{2}}{z^{4} f}\right] \rho_{x}=0, } \\
A_{t}^{\prime \prime} & +\left(\frac{1}{z}+\frac{f^{\prime}}{f}\right) A_{t}^{\prime}-\frac{2}{z^{2} f}\left(\frac{q \rho_{x}}{r_{s}}\right)^{2} A_{t}=0, \\
A_{\varphi}^{\prime \prime} & -\frac{1}{z} A_{\varphi}^{\prime}-\frac{2}{z^{2} f}\left(\frac{q \rho_{x}}{r_{s}}\right)^{2} A_{\varphi}=0,
\end{aligned}
$$

where the prime denotes the derivative with respect to $z$, and $f(z)$ is denoted by

$f=\frac{1}{2 \alpha z^{2}}\left[1-\sqrt{1-4 \alpha\left(1-z^{4}\right)}\right]$.

At the critical chemical potential $\mu_{c}$, the vector field $\rho_{x}$ is zero. So the equation of motion for the gauge field component $A_{t}$ turns out to be

$A_{t}^{\prime \prime}+\left(\frac{1}{z}+\frac{f^{\prime}}{f}\right) A_{t}^{\prime}=0$.

The above equation can not be solved directly, but considering the GB factor $\alpha$ is small, we can still get the effective general solution

$$
\begin{aligned}
A_{t}= & \mu+\frac{1}{4} c_{1}\left[\frac{2 \sqrt{\alpha} \arctan \left(\frac{\sqrt{\alpha} z^{2}}{\sqrt{-1-\alpha}}\right)}{\sqrt{-1-\alpha}}\right. \\
& \left.-\ln \left(1-z^{2}\right)+\ln \left(1+z^{2}\right)\right] .
\end{aligned}
$$

In order to keep $A_{t}$ finite, we have to fix $c_{1}=0$ in view of the Neumann-like boundary condition for $A_{t}$ at the tip of the soliton. Thus, it leads to that the physical solution of $A_{t}$ is a constant $\mu$ at the phase transition point.

Similarly, the equation of the spatial component $A_{\varphi}$ in Eq. (15) simplifies to

$A_{\varphi}^{\prime \prime}-\frac{1}{z} A_{\varphi}^{\prime}=0$ and the solution is

$A_{\varphi}=S_{\varphi}\left(1-z^{2}\right)$,

which fulfills the condition $A_{\varphi}(1)=0$ given in Eq. (11).

Now we consider the case that the chemical potential is away from (but very close to) the critical value $\mu_{c}$, the field equation for the vector field $\rho_{x}$ approaches to

$$
\begin{aligned}
\rho_{x}^{\prime \prime} & +\left(\frac{1}{z}+\frac{f^{\prime}}{f}\right) \rho_{x}^{\prime} \\
+ & {\left[\frac{1}{z^{2} f}\left(\frac{q \mu}{r_{s}}\right)^{2}-\frac{\left(1-z^{2}\right)^{2}}{z^{4} f^{2}}\left(\frac{q S_{\varphi}}{r_{s}}\right)^{2}-\frac{m^{2}}{z^{4} f}\right] \rho_{x}=0 . }
\end{aligned}
$$

From the boundary condition given in (12), we suppose that $\rho_{x}$ takes the form

$\rho_{x}(z) \sim \frac{\left\langle O_{x}\right\rangle}{r_{s}^{\Delta}} z^{\Delta} F(z)$,

where we have defined a trial function $F(z)$ with the boundary conditions $F(0)=1$ and $F^{\prime}(0)=0$, and $\Delta=1+$ $\sqrt{1+m^{2} L_{e f f}^{2}}$. Then, Eq. (21) can be rewritten as

$$
\begin{aligned}
& F^{\prime \prime}(z)+\left(\frac{2 \Delta+1}{z}+\frac{f^{\prime}}{f}\right) F^{\prime}(z) \\
& +\left[\frac{\Delta}{z}\left(\frac{\Delta}{z}+\frac{f^{\prime}}{f}\right)+\frac{1}{z^{2} f}\left(\frac{q \mu}{r_{s}}\right)^{2}\right. \\
& \left.-\frac{\left(1-z^{2}\right)^{2}}{z^{4} f^{2}}\left(\frac{q S_{\varphi}}{r_{s}}\right)^{2}-\frac{m^{2}}{z^{4} f}\right] F(z)=0 .
\end{aligned}
$$

Here we introduce a new parameter proposed in Ref. [53]

$K=\frac{q S_{y}}{r_{s}}$,

which can help to prevent the problem of the divergent behavior for the larger values of the parameter $k=\frac{S_{\varphi}}{\mu_{c}}$ that we had introduced in our previous work [38].

Following the Sturm-Liouville eigenvalue problem, the equation of motion for $F(z)$ can be converted into a standard Sturm-Liouville form

$\left(P F^{\prime}\right)^{\prime}+P\left[V+W\left(\frac{q \mu}{r_{s}}\right)^{2}\right] F=0$,

with

$$
\begin{aligned}
& P=\frac{z^{2 \Delta-1}\left[1-\sqrt{1-4 \alpha\left(1-z^{4}\right)}\right]}{2 \sqrt{\alpha}}, \\
& V=\frac{\Delta}{z}\left(\frac{\Delta}{z}+\frac{f^{\prime}}{f}\right)-\frac{m^{2}}{z^{4} f}-\frac{K^{2}\left(1-z^{2}\right)^{2}}{z^{4} f^{2}}, \\
& W=\frac{1}{z^{2} f} .
\end{aligned}
$$


Table 1 The critical value of chemical potential $\Lambda_{c}=q \mu_{c} / r_{s}$ of the holographic p-wave superfluid model obtained by the analytical S-L method (left column) and numerical calculation (right column) with a number of superfluid velocity $K=q S_{\varphi} / r_{S}$ and the GB coupling constant $\alpha=-0.19,0.0001,0.09$, respectively, for the fixed mass of the vector field $m^{2} L_{e f f}^{2}=-3 / 4$

\begin{tabular}{|c|c|c|c|c|c|c|}
\hline$\alpha$ & -0.19 & & 0.0001 & & 0.09 & \\
\hline$K=0.00$ & 1.62213 & 1.62201 & 1.73955 & 1.73888 & 1.82124 & 1.82057 \\
\hline$K=0.25$ & 1.62945 & 1.62932 & 1.74546 & 1.74476 & 1.82631 & 1.82580 \\
\hline$K=0.50$ & 1.65115 & 1.65116 & 1.76302 & 1.76374 & 1.84157 & 1.84123 \\
\hline$K=0.75$ & 1.68643 & 1.68667 & 1.79174 & 1.79174 & 1.86661 & 1.86638 \\
\hline$K=1.00$ & 1.73410 & 1.73473 & 1.83088 & 1.83112 & 1.90087 & 1.90090 \\
\hline
\end{tabular}

Table 2 The critical chemical potential $\Lambda_{c}=q \mu_{c} / r_{s}$ obtained by the analytical S-L method (left column) and numerical computation (right column) for the holographic p-wave superfluid model for different val-

\begin{tabular}{|c|c|c|c|c|c|c|}
\hline$\alpha$ & -0.19 & & 0.0001 & & 0.09 & \\
\hline$K=0.00$ & 2.11529 & 2.11349 & 2.26711 & 2.26533 & 2.37252 & 2.37071 \\
\hline$K=0.25$ & 2.11986 & 2.11826 & 2.27080 & 2.26890 & 2.37573 & 2.37378 \\
\hline$K=0.50$ & 2.13346 & 2.13211 & 2.28183 & 2.28017 & 2.38533 & 2.38344 \\
\hline$K=0.75$ & 2.15582 & 2.15483 & 2.30000 & 2.29859 & 2.40117 & 2.39955 \\
\hline$K=1.00$ & 2.18578 & 2.18578 & 2.32506 & 2.32377 & 2.42307 & 2.42167 \\
\hline
\end{tabular}

ues of $K$. We choose the GB coupling constant $\alpha=-0.19,0.0001$, 0.09 , respectively, and fix the mass of the vector field by $m^{2} L_{\text {eff }}^{2}=0$

Table 3 The critical chemical potential $\Lambda_{c}=q \mu_{c} / r_{s}$ obtained from the S-L method (left column) and numerical calculation (right column) for the holographic p-wave superfluid model for chosen values of the GB factor $\alpha$ and various of $K$ with the fixed mass $m^{2} L_{e f f}^{2}=5 / 4$

\begin{tabular}{|c|c|c|c|c|c|c|}
\hline$\alpha$ & -0.19 & & 0.0001 & & 0.09 & \\
\hline$K=0.00$ & 2.60126 & 2.59927 & 2.78720 & 2.78503 & 2.91642 & 2.91419 \\
\hline$K=0.25$ & 2.60438 & 2.60258 & 2.78973 & 2.78756 & 2.91862 & 2.91644 \\
\hline$K=0.50$ & 2.61370 & 2.61203 & 2.79729 & 2.79526 & 2.92521 & 2.92306 \\
\hline$K=0.75$ & 2.62910 & 2.62752 & 2.80982 & 2.80797 & 2.93614 & 2.93410 \\
\hline$K=1.00$ & 2.65037 & 2.64939 & 2.82717 & 2.82565 & 2.95131 & 2.94939 \\
\hline
\end{tabular}

Therefore, we can obtain the minimum eigenvalue of $\Lambda=$ $q \mu / r_{s}$ which minimizes the following functional

$\Lambda^{2}=\left(\frac{q \mu}{r_{s}}\right)^{2}=\frac{\int_{0}^{1} P\left(F^{\prime 2}-V F^{2}\right) d z}{\int_{0}^{1} P W F^{2} d z}$.

To further estimate it, we assume the trial function to be $F(z)=1-a z^{2}$ with a constant $a$.

In Tables 1, 2 and 3, we present the critical chemical potential $\Lambda_{c}=\Lambda_{\text {min }}$, which results from the minimum eigenvalues of $\Lambda^{2}$, for different values of the GB factor $\alpha$ and the dimensionless parameter $K$ with the fixed mass of the vector field $m^{2} L_{e f f}^{2}=-3 / 4,0,5 / 4$, respectively. As an example, with the chosen $K=0.25$ and the fixed mass $m^{2} L_{e f f}^{2}=-3 / 4$, we get the minimum eigenvalue $\Lambda_{\min }^{2}=2.65512$ at $a=0.30832$ for $\alpha=-0.19$, which gives the critical chemical potential $\Lambda_{c}=1.62945$. We can observe that the analytical results derived from the $\mathrm{S}-\mathrm{L}$ method are greatly consistent with the numerical computations. This shows that the $\mathrm{S}-\mathrm{L}$ method is an effective analyti- 

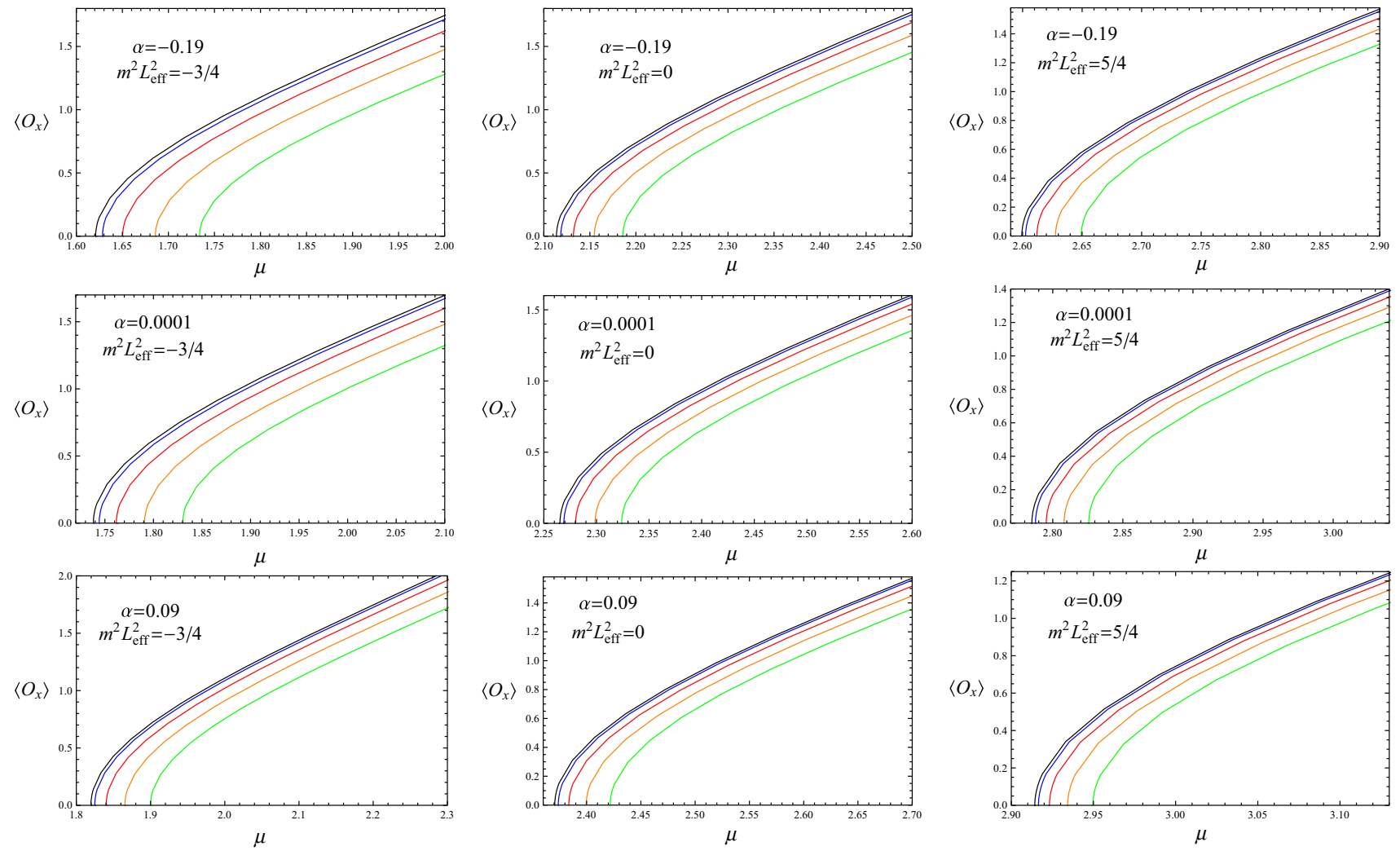

Fig. 1 The condensate as a function of the chemical potential for different values of Gauss-Bonnet parameter $\alpha$ with the fixed mass of the vector field $m^{2} L_{e f f}^{2}=-3 / 4,0,5 / 4$, respectively in the holographic p-wave superfluid model in AdS soliton. In each panel the five lines

to the larger critical chemical potential $\mu_{c}$ with $\alpha$ and the mass fixed. The similar property holds in the p-wave superfluid model in Einstein-Maxwell theory [38].

\subsection{Critical phenomena}

In this subsection we move on to investigate the condensation of the vector operator and the relation between the charge density and the chemical potential, which can help us further understand the properties of the p-wave superfluid phase transition in GB AdS soliton background.

Near the critical point, i.e., $\mu \rightarrow \mu_{c}$, the vector field $\rho_{x}$ approximates to (22), so the equation of motion for $A_{t}$ presents

$A_{t}^{\prime \prime}+\left(\frac{1}{z}+\frac{f^{\prime}}{f}\right) A_{t}^{\prime}-\frac{2 z^{2(\Delta-1)}}{f}\left(\frac{q\left\langle O_{x}\right\rangle}{r_{s}^{\Delta+1}}\right)^{2} F^{2} A_{t}=0$.

It should be noted that the condensation of the vector operator $\left\langle O_{x}\right\rangle$ is very small when $\mu \rightarrow \mu_{c}$, so we are able to expand $A_{t}(z)$ in $\left\langle O_{x}\right\rangle$ as follow

$A_{t}(z) \sim \mu_{c}+\left\langle O_{x}\right\rangle \chi(z)+\cdots$. from left to right correspond to increasing superfluid velocity, i.e., $K=q S_{\varphi} / r_{s}=0$ (black), 0.25 (blue), 0.50 (red), 0.75 (orange) and 1.00 (green) respectively. We scale $q=1$ and $r_{s}=1$ in the numerical calculation

We have to impose $\chi(1)=0$ and $\chi^{\prime}(1)=$ constant to recover the boundary condition at the tip. For simplicity, the following function is introduced

$\chi(z)=\mu_{c} \frac{2 q^{2}\left\langle O_{x}\right\rangle}{r_{s}^{2(\Delta+1)}} \xi(z)$,

then using Eqs. (28) and (29), we can easily arrive at the equation of motion for $\xi(z)$

$\left(Q \xi^{\prime}\right)^{\prime}-\frac{z^{2(\Delta-1)} Q}{f} F^{2}=0$,

with

$Q(z)=\frac{1-\sqrt{1-4 \alpha\left(1-z^{4}\right)}}{2 \sqrt{\alpha} z}$.

Considering the asymptotic behavior of $A_{t}$ in Eq. (12) and expanding $\xi(z)$ near the boundary $z=0$, we get

$$
\begin{aligned}
& A_{t}(z) \simeq \mu-\frac{\rho}{r_{s}^{2}} z^{2} \simeq \mu_{c} \\
& \quad+2 \mu_{c}\left(\frac{q\left\langle O_{x}\right\rangle}{r_{s}^{\Delta+1}}\right)^{2}\left[\xi(0)+\xi^{\prime}(0) z+\frac{1}{2} \xi^{\prime \prime}(0) z^{2}+\cdots\right] .
\end{aligned}
$$



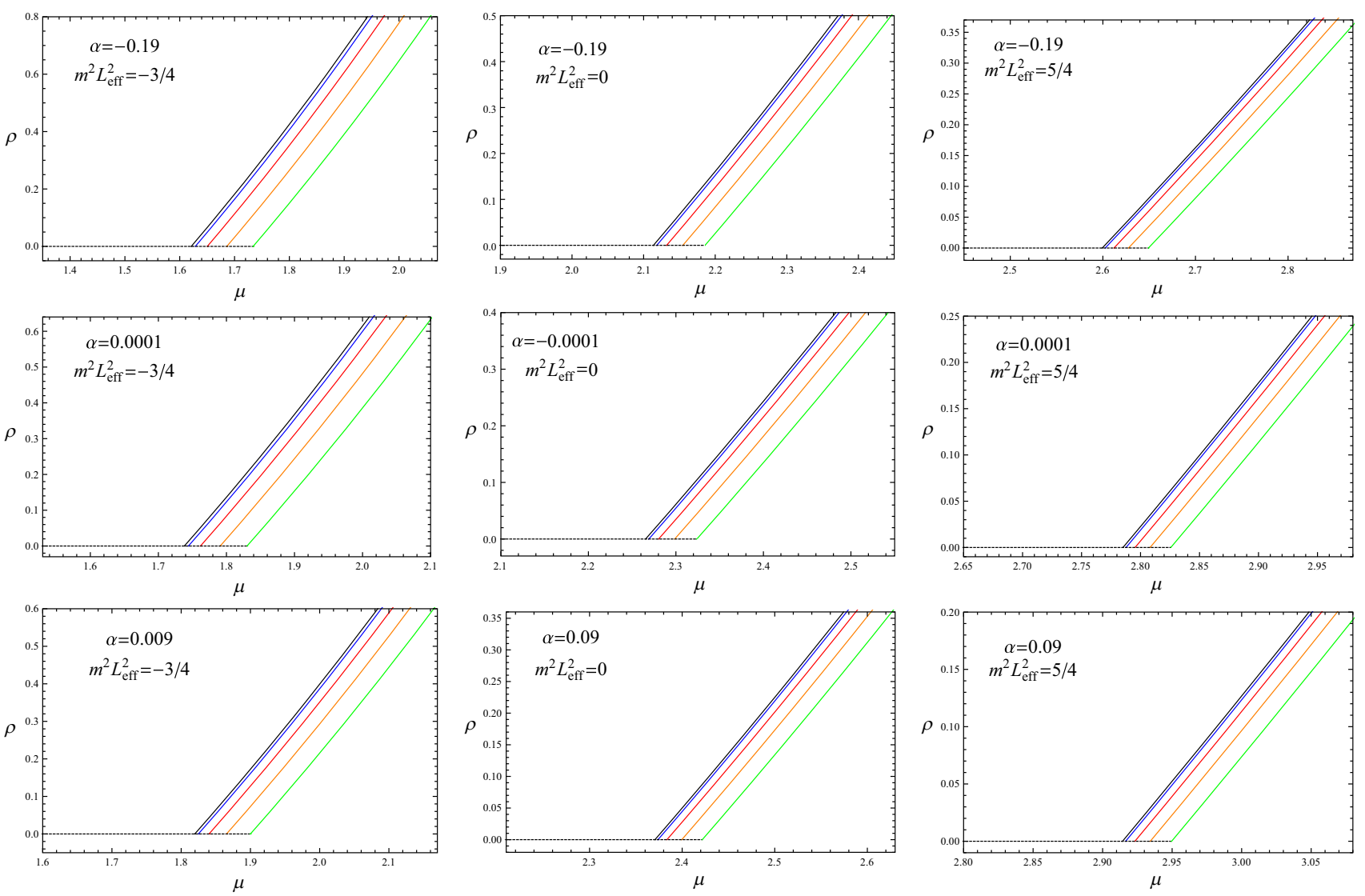

Fig. 2 The charge density as a function of the chemical potential for different values of Gauss-Bonnet parameter $\alpha$ with the fixed mass of the vector field $m^{2} L_{e f f}^{2}=-3 / 4,0,5 / 4$, respectively in the holographic pwave superfluid model in AdS soliton. In each panel the five lines from left to right correspond to increasing superfluid velocity, i.e., $K=0$ (black), 0.25 (blue), 0.50 (red), 0.75 (orange) and 1.00 (green) respectively. We scale $q=1$ and $r_{s}=1$ in the numerical calculation

represents the second-order phase transition, with the critical exponent of system taking the mean-field value $1 / 2$. Moreover, both the GB coupling constant $\alpha$ and the spatial component of the gauge field do not have any effect on the second-order phase transition. Figure 1 illustrates that there is a second-order phase transition when the chemical potential $\mu$ arrives at the critical value $\mu_{c}$, and the AdS soliton reaches the superconductor (or superfluid) phase for larger $\mu$, which confirms the above analytical conclusion. Also we note that for the vanishing superfluid velocity, the situation is the same as the one discussed in Ref. [29].

On the other hand, from the coefficients of the $z^{2}$ term in Eq. (33), we know that the dependence of the charge density $\rho$ on the chemical potential $\mu$ reads

$\frac{\rho}{r_{s}^{2}}=-\left(\frac{q\left\langle O_{x}\right\rangle}{r_{s}^{\Delta+1}}\right)^{2} \mu_{c} \xi^{\prime \prime}(0)$.

coupling constant $\alpha$, the order parameter $\left\langle O_{x}\right\rangle$ always yields

$\left\langle O_{x}\right\rangle \sim\left(\mu-\mu_{c}\right)^{1 / 2}$,

which reveals that in the GB AdS soliton background, the phase transition of the holographic p-wave superfluid model
From the integration of both sides of Eq. (31) and the fact that $\xi^{\prime}(0)=0$ revealed by the comparison of the coefficients of the $z^{1}$ term in Eq. (33), we have 
$\xi^{\prime \prime}(0)=\left.\left[\frac{\xi^{\prime}(z)}{z}\right]\right|_{z \rightarrow 0}=-\frac{2 \sqrt{\alpha}}{1-\sqrt{1-4 \alpha}} \int_{0}^{1} \frac{z^{2(\Delta-1)} Q}{f} F^{2} d z$.

Then, inserting the above equation into Eq. (37), we attain

$\frac{\rho}{r_{s}^{2}}=\Gamma(\alpha, K, m)\left(\mu-\mu_{c}\right)$,

where $\Gamma(\alpha, K, m)$ is given by

$\Gamma(\alpha, K, m)=\frac{\sqrt{\alpha}}{(1-\sqrt{1-4 \alpha}) \xi(0)} \int_{0}^{1} \frac{z^{2(\Delta-1)} Q F^{2}}{f} d z$.

The charge density $\rho$ for the holographic p-wave superfluid depends on the GB factor $\alpha$, the parameter $K$ and the mass of the vector field $m^{2}$ via function $\Gamma$, and it is proportional to $\left(\mu-\mu_{c}\right)$. As an example, for the case of $K=0.25$ and $m^{2} L_{\text {eff }}^{2}=5 / 4$, from Eq. (39) we have $\rho=1.01646\left(\mu-\mu_{c}\right)$ with $\alpha=0.0001$. The relation between the charge density and the chemical potential $\rho \sim\left(\mu-\mu_{c}\right)$ derived from the analytical method backs up the numerical results shown in Fig. 2.

\section{3 $A_{\varphi}$ in holographic p-wave superfluid}

Let us remind that the vector field approximates to $\rho_{x} \sim$ $\frac{\left\langle O_{x}\right\rangle}{r_{s} \Delta_{+}} z^{\Delta_{+}} F(z)$ near the critical point, so the equation of motion for the spatial component $A_{\varphi}$ of the vector potential can be rewritten as

$A_{\varphi}^{\prime \prime}-\frac{1}{z} A_{\varphi}^{\prime}-\frac{2 z^{2(\Delta-1)}}{f}\left(\frac{q\left\langle O_{x}\right\rangle}{r_{s}^{\Delta+1}}\right)^{2} F^{2} A_{\varphi}=0$,

where $F(z)$ obeys $F(0)=1$ and $F^{\prime}(0)=1$. Similarly to the procedure in the preceding subsection, as $\mu \rightarrow \mu_{c}, A_{\varphi}$ can be expanded in small $\left\langle O_{x}\right\rangle$ as

$A_{\varphi}(z) \sim S_{\varphi}\left(1-z^{2}\right)+\left\langle O_{x}\right\rangle w(z)+\cdots$.

From Eqs. (41) and (42), we easily find that the introduced function $w(z)$ satisfies the following equation:

$w^{\prime \prime}-\frac{1}{z} w^{\prime}=S_{\varphi} \frac{q^{2}\left\langle O_{x}\right\rangle}{r_{s}^{2(\Delta+1)}} z^{2(\Delta-1)} \frac{2\left(1-z^{2}\right) F^{2}}{f}$.

We can expand $w(z)$ close to the boundary $z=0$ as

$w(z)=w(0)+w^{\prime}(0) z+\frac{1}{2} w^{\prime \prime}(0) z^{2}+\cdots$.

Substituting the above expansion into Eq. (42), and comparing with the boundary condition (12) for $A_{\varphi}$, from the $z^{2}$ term, we can deduce that the superfluid current reads

$\frac{J_{\varphi}}{r_{s}^{2}}=S_{\varphi}+S_{\varphi}\left(\frac{q\left\langle O_{x}\right\rangle}{r_{s}^{\Delta+1}}\right)^{2} \int_{0}^{1} z^{2(\Delta-1)} \frac{\left(1-z^{2}\right) F^{2}}{f} d z$.

This expression shows that, in soliton background, GB coupling constant $\alpha$ does not directly affect the current but through $\mu_{c}(\alpha)$ due to the relation $\left\langle O_{x}\right\rangle \sim\left(\mu-\mu_{c}\right)^{1 / 2}$.
Eventually the behavior of $A_{\varphi}$ near the critical point can be described as

$$
\begin{aligned}
A_{\varphi}= & S_{\varphi}\left(1-z^{2}\right) \\
& -S_{\varphi}\left(\frac{q\left\langle O_{x}\right\rangle}{r_{s}^{\Delta+1}}\right)^{2} z^{2} \int_{0}^{1} x^{2(\Delta-1)} \frac{\left(1-x^{2}\right) F^{2}}{f} d x
\end{aligned}
$$

which matches the behavior of $A_{\varphi}$ in Eq. (20) at the critical chemical potential $\mu_{c}$.

\section{Holographic p-wave superfluid model in Gauss-Bonnet AdS black hole}

The holographic p-wave model of superfluidity in AdS soliton background is used to describe a system at zero temperature. In this section we employ the S-L method to analytically investigate the holographic $\mathrm{p}$-wave superfluid model with a certain temperature. For this purpose, our attention is concentrated on the Maxwell complex vector field model with the the same action of matter as Eq. (6), and taking into account the background of five-dimensional AdS black hole in the GB gravity

$d s^{2}=-f(r) d t^{2}+\frac{d r^{2}}{f(r)}+r^{2}\left(d x^{2}+d y^{2}+d z^{2}\right)$,

where $f(r)=\frac{r^{2}}{2 \alpha}\left[1-\sqrt{1-\frac{4 \alpha}{L^{2}}\left(1-\frac{r_{+}^{4}}{r^{4}}\right)}\right]$, in units in which the AdS radius is unity, i.e., $L=1$. The Hawking temperature of the black hole is related to the horizon radius $r_{+}$as $T_{B H}=\frac{r_{+}}{\pi}$. We suppose that the gauge field has the form $A=A_{t}(r) d t+A_{y}(r) d y$ and the vector field presents $\rho_{\mu} d x^{\mu}=\rho_{x}(r) d x$. Then we have the field equations

$$
\begin{aligned}
& \rho_{x}^{\prime \prime}+\left(\frac{f^{\prime}}{f}+\frac{1}{r}\right) \rho_{x}^{\prime}+\left(\frac{q^{2} A_{t}^{2}}{f^{2}}-\frac{q^{2} A_{y}^{2}}{r^{2} f}-\frac{m^{2}}{f}\right) \rho_{x}=0 \\
& A_{t}^{\prime \prime}+\frac{3}{r} A_{t}^{\prime}-\frac{2 q^{2} \rho_{x}^{2}}{r^{2} f} A_{t}=0 \\
& A_{y}^{\prime \prime}+\left(\frac{f^{\prime}}{f}+\frac{1}{r}\right) A_{y}^{\prime}-\frac{2 q^{2} \rho_{x}^{2}}{r^{2} f} A_{y}=0
\end{aligned}
$$

where the prime denotes the derivative with respect to $r$.

Imposing the regularity conditions at the horizon $r=r_{+}$, we have

$$
\begin{aligned}
A_{t}\left(r_{+}\right) & =0, \quad \rho_{x}^{\prime}\left(r_{+}\right) \\
& =\frac{1}{f^{\prime}\left(r_{+}\right)}\left[\frac{q^{2} A_{y}^{2}\left(r_{+}\right)}{r_{+}^{2}}+m^{2}\right] \rho_{x}\left(r_{+}\right), \\
A_{y}^{\prime} & =\frac{2 q^{2} \rho_{x}^{2}\left(r_{+}\right)}{r_{+}^{2} f^{\prime}\left(r_{+}\right)} A_{y}\left(r_{+}\right) .
\end{aligned}
$$

It should be noted that $A_{t}$ is vanish at $r=r_{+}$, which is in strong contrast to the case in AdS soliton where time compo- 
nent $A_{t}\left(r_{s}\right)$ of the the vector potential is a nonzero constant at the tip. On the boundary regime where $r \rightarrow \infty$, the asymptotic solutions of the matter fields are the same as the ones given in Eq. (12) by replacing $A_{\varphi}, S_{\varphi}$ and $J_{\varphi}$ with $A_{y}, S_{y}$ and $J_{y}$, respectively. Also, as mentioned in the previous section, we focus on the dual operator $\left\langle O_{x}\right\rangle=\rho_{x}$ and set the boundary condition $\rho_{x-}=0$.

There are still some useful scaling symmetries for the metric, bulk matter fields and the equations of the motion, they yield

$r \rightarrow \lambda r, \quad(t, x, y, z) \rightarrow \frac{1}{\lambda}(t, x, y, z)$,

$q \rightarrow q, \quad\left(\rho_{x}, A_{t}, A_{y}\right) \rightarrow \lambda\left(\rho_{x}, A_{t}, A_{y}\right)$,

which also give the related transformation of the physical quantities

$$
\begin{aligned}
& \left(T, \mu, S_{y}\right) \rightarrow \lambda\left(T, \mu, S_{y}\right), \quad\left(\rho, J_{y}\right) \rightarrow \lambda^{3}\left(\rho, J_{y}\right), \\
& \rho_{x+} \rightarrow \lambda^{\Delta_{+}+1} \rho_{x+} .
\end{aligned}
$$

The scaling symmetries enable us to fix $q=1$ and $r_{+}=1$ when performing numerical calculations and checking the analytical expressions in this section.

It will be convenient to rewrite the equations of motion of the fields in $z=r_{+} / r$-coordinates as

$$
\begin{aligned}
& \rho_{x}^{\prime \prime}+\left(\frac{f^{\prime}}{f}+\frac{1}{z}\right) \rho_{x}^{\prime} \\
& +\left[\frac{1}{z^{4} f^{2}}\left(\frac{q A_{t}}{r_{+}}\right)^{2}-\frac{1}{z^{2} f}\left(\frac{q A_{y}}{r_{+}}\right)^{2}-\frac{m^{2}}{z^{4} f}\right] \rho_{x}=0 \\
& A_{t}^{\prime \prime}-\frac{1}{z} A_{t}^{\prime}-\frac{2 q^{2} \rho_{x}^{2}}{r_{+}^{2} z^{2} f} A_{t}=0 \\
& A_{y}^{\prime \prime}+\left(\frac{f^{\prime}}{f}+\frac{1}{z}\right) A_{y}^{\prime}-\frac{2 q^{2} \rho_{x}^{2}}{r_{+}^{2} z^{2} f} A_{y}=0
\end{aligned}
$$

where now the prime denotes the derivative with respect to $z$, and $f(z)$ is the same as the one defined in Eq. (16).

Before going further, we would like to give a comment. For the holographic p-wave model of superfluidity in the background of AdS black hole, the transition between the superfluid and the normal phase will switch from the second order to the first order in the large value of the superfluid velocity. Particularly, the phase transition of the system always belongs to the second order either when the superfluid velocity is small or the mass of the vector field is sufficiently large. To facilitate our analytical discussion, in this section we consider the later situation, and in the following computation we fix the large mass of the vector field by $m^{2} L_{e f f}^{2}=3$ where the superfluid phase transition is of the second order.

\subsection{Critical temperature for $\mathrm{p}$-wave superfluid}

When $T \geq T_{c}$, the vector field nearly vanishes, i.e., $\rho_{x} \rightarrow 0$, so the field equation for $A_{t}$ reduces to
$A_{t}^{\prime \prime}-\frac{1}{z} A_{t}^{\prime}=0$

The general solution for the above equation reads $A_{t}=c_{1}+$ $c_{2} z^{2}$, with the boundary condition $A_{t}(1)=0$. Then we arrive at

$A_{t} \simeq \frac{\lambda r_{+}}{q}\left(1-z^{2}\right)$

where we have set $\lambda=\frac{q \mu}{r_{+c}}$ with $r_{+c}$ stands for the horizon radius for the black hole at the critical temperature $T_{c}$.

Likewise, above the critical point the spatial component $A_{y}$ satisfies the following equation

$A_{y}^{\prime \prime}+\left(\frac{f^{\prime}}{f}+\frac{1}{z}\right) A_{y}^{\prime}=0$.

For small GB factor $\alpha$, we readily get the physical solutions $A_{y}=S_{y}$ from the Neumann-like boundary condition.

Thus, for $T$ slightly below the critical value, the equation of motion for $\rho_{x}$ presents

$$
\begin{aligned}
& \rho_{x}^{\prime \prime}+\left(\frac{f^{\prime}}{f}+\frac{1}{z}\right) \rho_{x}^{\prime} \\
& +\left[\frac{\left(1-z^{2}\right)^{2}}{z^{4} f^{2}} \lambda^{2}-\frac{1}{z^{2} f}\left(\frac{q S_{y}}{r_{+}}\right)^{2}-\frac{m^{2}}{z^{4} f}\right] \rho_{x}=0 .
\end{aligned}
$$

Considering the asymptotic behavior of $\rho_{x}$ near the boundary $z \rightarrow 0$, we can write the approximate expression

$\rho_{x}(z) \sim \frac{\left\langle O_{x}\right\rangle}{r_{+}^{\Delta}} z^{\Delta} F(z)$,

where the conformal dimension of the vector operator $\Delta=$ $1+\sqrt{1+m^{2} L_{e f f}^{2}}$. In order to further improve the agreement with the numerical results, we assume that $F(z)=$ $1-a z^{2}+b z^{3}$ which is slightly different from the one in previous section, and it has to fulfill the constraints $F(0)=1$ and $F^{\prime}(0)=0$. Eventually, by substituting Eq. (57) into Eq. (56), we attain the euqation of motion for $F(z)$ in a standard Sturm-Liouville form

$\left(P F^{\prime}\right)^{\prime}+P\left(U+\lambda^{2} R\right) F=0$,

with

$U=\frac{\Delta}{z}\left(\frac{\Delta}{z}+\frac{f^{\prime}}{f}\right)-\frac{m^{2}}{z^{4} f}-\frac{K^{2}}{z^{2} f}, \quad R=\frac{\left(1-z^{2}\right)^{2}}{z^{4} f}$,

where $P$ has been defined in Eq. (26) and $K=\frac{q S_{y}}{r_{+}}$. In the light of the Sturm-Liouville eigenvalue problem, the minimum eigenvalue of the parameter $\lambda^{2}=\frac{q^{2} \mu^{2}}{r_{+c}^{2}}$ can be estimated from the variation of the following expression

$\lambda^{2}=\frac{\int_{0}^{1} P\left(F^{\prime 2}-U F^{2}\right) d z}{\int_{0}^{1} P R F^{2} d z}$. 
Table 4 The critical temperature $T_{c} / q \mu$ of the holographic p-wave superfluid model obtained by the analytical S-L method (left column) and by numerical calculation (right column), with different values of the superfluid velocity $K=q S_{y} / r_{+}$and the GB coupling constant $\alpha=-0.19,0.0001,0.09$, respectively, for the fixed mass $m^{2} L_{e f f}^{2}=3$

\begin{tabular}{|c|c|c|c|c|c|c|}
\hline$\alpha$ & -0.19 & & 0.0001 & & 0.09 & \\
\hline$K=0.00$ & 0.05707 & 0.05736 & 0.04973 & 0.04977 & 0.04516 & 0.04532 \\
\hline$K=0.25$ & 0.05693 & 0.05722 & 0.04946 & 0.04967 & 0.04507 & 0.04523 \\
\hline$K=0.50$ & 0.05651 & 0.05680 & 0.04914 & 0.04935 & 0.04480 & 0.04496 \\
\hline$K=0.75$ & 0.05583 & 0.05613 & 0.04862 & 0.04897 & 0.04437 & 0.04453 \\
\hline$K=1.00$ & 0.05493 & 0.05525 & 0.04793 & 0.04815 & 0.04379 & 0.04396 \\
\hline
\end{tabular}

The parameter $\lambda_{\min }=\frac{q \mu}{r_{+c}}$ gives the value of horizon radius $r_{+}$. Since the analysis is valid close to the transition point $T_{C} \sim T_{B H}$, using the definition of the Hawking temperature $T_{B H}$ of the black hole, we can compute the critical temperature of the superconductor by the following relation

$$
\frac{T_{c}}{q \mu}=\frac{1}{\pi \lambda_{\min }} \text {. }
$$

As an example, for the case of $K=0.25$ and $m^{2} L_{\text {eff }}^{2}=3$, with the help of Eq. (61) we have the critical temperature $T_{c}=0.04507$ at $a=2.46232$ and $b=1.58561$ corresponding to $\alpha=0.09$, and $T_{c}=0.05693$ at $a=2.55498$ and $b=1.66813$ corresponding to $\alpha=-0.19$. In Table 4 , we exhibit the typical values of the critical temperature $T_{c}$ which depends on the GB coupling constant $\alpha$ and the superfluid parameter $K$ for the case of the fixed mass of the vector field $m^{2} L_{e f f}^{2}=3$. We can clearly see that the critical temperature $T_{c}$ diminishes as the GB parameter $\alpha$ increases for various of $K$. That is to say, the higher curvature correction will make the condensation of the vector operator harder to be formed. In addition, for the same strength of the curvature correction, the critical temperature $T_{c}$ decreases when the superfluid velocity $K$ becomes bigger, which backs up the numerical findings in Ref. [45]. Furthermore, the comparison with the numerical findings indicates that the consistency of our analytical results derived from the $\mathrm{S}-\mathrm{L}$ method with the ones from numerical calculation is impressive.

\subsection{Condensation values}

In this subsection, we aim to investigate the critical phenomena of the p-wave superfluid model in AdS black hole and figure out the correlation between the condensation operator and the GB coupling constant. For $T$ below but not far from the critical one $T_{c}$, we can expand the time component $A_{t}$ of the gauge field in small vector operator $\left\langle O_{x}\right\rangle$

$\frac{q A_{t}(z)}{r_{+}}=\lambda_{c}\left(1-z^{2}\right)+\left\langle O_{x}\right\rangle \kappa(z)+\cdots$,

with the boundary conditions $\kappa(1)=0$ resulting from $A_{t}(1)=0$ at the horizon. We redefine $\kappa(z)$ by a new function $\varsigma(z)$ as

$\kappa(z)=\frac{q^{2}\left\langle O_{x}\right\rangle}{r_{+}^{2(\Delta+1)}} \varsigma(z)$,

so near the critical temperature, we easily get the equation of motion for $\varsigma(z)$

$$
\left(\frac{\varsigma^{\prime}}{z}\right)^{\prime}=\frac{2 \lambda z^{2 \Delta-3}\left(1-z^{2}\right)}{f} F^{2} .
$$

Near the boundary $z=0$, from the asymptotic behavior of $A_{t}$ and the expansion $\varsigma(z)=\varsigma(0)+\varsigma^{\prime}(0) z+\frac{1}{2} \varsigma^{\prime \prime}(0) z^{2}+\cdots$, Eq. (62) can be rewritten as

$$
\begin{aligned}
\frac{q A_{t}}{r_{+}}= & \lambda\left(1-z^{2}\right) \approx \lambda_{c}\left(1-z^{2}\right) \\
& +\frac{q^{2}\left\langle O_{x}\right\rangle^{2}}{r_{+}^{2(\Delta+1)}}\left[\varsigma(0)+\varsigma^{\prime}(0) z+\frac{1}{2} \varsigma^{\prime \prime}(0) z^{2}+\cdots\right]
\end{aligned}
$$

Comparing the coefficients multiplying $z^{0}, z^{1}$ and $z^{2}$ in both sides of the above formula, respectively, we have

$$
\begin{aligned}
& \lambda=\lambda_{c}+\left\langle O_{x}\right\rangle \varsigma(0), \\
& \lambda=\lambda_{c}-\frac{1}{2}\left\langle O_{x}\right\rangle \varsigma^{\prime \prime}(0), \\
& \varsigma^{\prime}(0)=0 .
\end{aligned}
$$

Obviously, there is a relation $\varsigma(0)=-\varsigma^{\prime \prime}(0) / 2$ to make the above equations ture. Therefore, for the further calculation we need the value of $\varsigma^{\prime \prime}(0)$. On the other hand, we observe that the third part of Eq. (66) is consistent with the following relation by making integration of both sides of Eq. (64)

$\varsigma^{\prime \prime}(0)=\left.\left[\frac{\varsigma^{\prime}(z)}{z}\right]\right|_{z \rightarrow 0}=-\int_{0}^{1} \frac{2 \lambda z^{2 \Delta-3}\left(1-z^{2}\right)}{f} F^{2} d z$.

In virtue of Eqs. (61), (66) and (67), the correlation between the condensation value of the vector operator $\left\langle O_{x}\right\rangle$ and the temperature is given by

$q\left\langle O_{x}\right\rangle=T_{c}^{\Delta+1} \Upsilon \sqrt{1-\frac{T}{T_{c}}}$ 

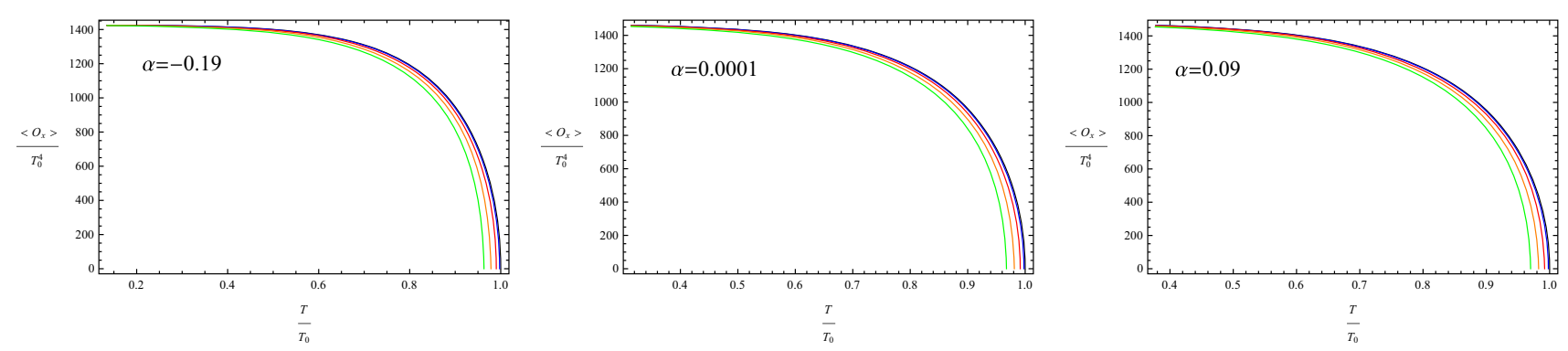

Fig. 3 The condensate as a function of the temperature for different values of GB parameter $\alpha$ with the fixed mass of the vector field $m^{2} L_{e f f}^{2}=3$ in the holographic p-wave superfluid model in AdS black hole. In each panel the five lines from top to bottom correspond to

where we have set

$\Upsilon=\pi^{\Delta+1}\left[\int_{0}^{1} \frac{1-z^{2}}{f} z^{2 \Delta-3} F^{2} d z\right]^{-1 / 2}$.

To concretely detect the condensation operator of p-wave superfluid model with respect to $T$ in GB AdS black hole with large mass of the vector field by numerical shooting method, we focus on the case of the mass fixed by $m^{2} L_{\text {eff }}^{2}=3$. In Fig. 3, we plot the condensate of the vector operator as a function of temperature for several values of the superfluid velocity $K=q S_{y} / r_{+}$with the fixed GB factor $\alpha=-0.19,0.0001,0.09$, respectively. We remark that in each panel, the curves for different $K$ are similar. In other words, although the coefficent $\Upsilon$ is different, near the critical point $T_{c}$, the condensation operator $\left\langle O_{x}\right\rangle$ always takes the form

$\left\langle O_{x}\right\rangle \sim\left(1-T / T_{C}\right)^{1 / 2}$,

which implies that the phase transition of the holographic p-wave superfluid model belongs to the second order and the critical exponent of the system takes the mean-field value $1 / 2$ for the case of the fixed mass $m^{2} L_{\text {eff }}^{2}=3$ in AdS black hole background. This result is independent of the GB coupling constant $\alpha$. Moreover, we see that for the fixed value of the superfluid velocity $K$, the critical temperature decreases when the GB factor $\alpha$ grows. Again, it states that the higher curvature correction makes the condensation of the vector operator harder to occur.

3.3 $A_{y}$ in holographic p-wave superfluid in AdS black hole

Having in mind that the vector field $\rho_{x}(z)=\frac{\left\langle O_{x}\right\rangle}{r_{+}^{\Delta}} z^{\Delta} F(z)$ when $T$ tends to $T_{c}$. The spatial component $A_{y}(z)$ satisfies the equation of motion in the form

$A_{y}^{\prime \prime}+\left(\frac{1}{z}+\frac{f^{\prime}}{f}\right) A_{y}^{\prime}-\frac{2 z^{2(\Delta-1)}}{f}\left(\frac{q\left\langle O_{x}\right\rangle}{r_{+}^{\Delta+1}}\right)^{2} F^{2} A_{y}=0$. increasing superfluid velocity, i.e., $K=q S_{y} / r_{+}=0$ (black), 0.25 (blue), 0.50 (red), 0.75 (orange) and 1.00 (green), respectively. We scale $q=1$ and $r_{+}=1$ in the numerical calculation

Near the critical point, $A_{y}$ takes the approximation

$A_{y} \sim S_{y}+\left\langle O_{x}\right\rangle \tau(z)+\cdots$.

Inserting Eq. (72) into Eq. (71), the equation of motion for $\tau(z)$ is given by

$\left(Q \tau^{\prime}\right)^{\prime}=S_{y} \frac{q^{2}\left\langle O_{x}\right\rangle}{r_{+}^{2(\Delta+1)}} \frac{2 z^{2(\Delta-1)} Q}{f} F^{2}$,

where $Q(z)$ has been introduced in Eq. (32). As we processed in the preceding subsection, the expansion of $\tau(z)$ near $z=0$ presents

$\tau(z)=\tau(0)+\tau^{\prime}(0) z+\frac{1}{2} \tau^{\prime \prime}(0) z^{2}+\cdots$.

Substituting the expansion into Eq. (72) and comparing the coefficient of the $z^{1}$ term with the one in the asymptotic behavior of $A_{y}$ on the boundary, we have $\tau^{\prime}(0) \rightarrow 0$. By virtue of this condition and integrating both sides of Eq. (73), the following relation is satisfied

$$
\tau^{\prime \prime}(0)=\left.\frac{\tau^{\prime}(z)}{z}\right|_{z \rightarrow 0}=-S_{y} \frac{q^{2}\left\langle O_{x}\right\rangle}{r_{+}^{2(\Delta+1)}} \frac{2 \sqrt{\alpha}}{1-\sqrt{1-4 \alpha}} \int_{0}^{1} \frac{2 z^{2(\Delta-1)} Q}{f} F^{2} d z .
$$

Consequently, we get

$$
\begin{aligned}
A_{y}= & S_{y}-S_{y} \\
& \times\left(\frac{q\left\langle O_{x}\right\rangle}{r_{+}^{\Delta+1}}\right)^{2} \frac{2 \sqrt{\alpha}}{1-\sqrt{1-4 \alpha}} z^{2} \int_{0}^{1} \frac{z^{2(\Delta-1)} Q}{f} F^{2} d z,
\end{aligned}
$$

where the term multiplying $z^{2}$ stands for the superfluid current. In view of the dependence of $\left\langle O_{x}\right\rangle$ on the temperature, from Eq. (70), we can easily find that the current $J_{y}$ depends on the GB coupling constant $\alpha$ indirectly via $T_{c}(\alpha)$, and there is a linear relation between the current and temperature, i.e., $J_{y} \propto\left(1-\frac{T}{T_{c}}\right)$. 


\section{Conclusions}

In this paper, we have analytically investigated the general properties of holographic p-wave model of superfluid in Gauss-Bonnet gravity by employing the Sturm-Liouville eigenvalue problem. We performed our analysis in the background of AdS soliton as well as AdS black hole in the probe limit and deduced the dependence of the critical chemical potential and the critical temperature on the GB coupling constant $\alpha$, which are verified by numerical calculation and in good agreement with the numerical findings. Our results have shown that: for the case in AdS soliton with different mass of the vector field, the critical chemical potential increases with the increase of the GB coupling constant $\alpha$, while for the case in AdS black hole with the larger mass of vector field, the critical temperature decreases for the larger GB factor. Both of the observations reveal the fact that the higher curvature correction hinders the formation of the condensation of the vector operator in holographic $p$-wave superfluid models. On the other hand, based on the S-L method, we attain the condensation of the vector operator and the charge density with respect to the chemical potential in soliton case, the relation between the condensation value and temperature in black hole case and the behavior of the spatial component of the gauge field near the phase transition point for the p-wave superfluid model. In the AdS soliton background, the superfluid phase transition is always of the second order and the critical exponent of the condensation operator takes the mean-field value $1 / 2$, which cannot be affected by the GB coupling constant $\alpha$. Especially, we analytically demonstrate that, for the p-wave superfluid model in AdS black hole, when the mass of the vector field is sufficiently large, the phase transition always belongs to the second order for different values of superfluid velocity, that is to say, the spatial component of the gauge field modeling the superfluid cannot bring up the first-order phase transition. And this conclusion is independent of the GB parameter. We also carry out a numerical study of the condensation of the vector operator by using the shooting method. All the analytical results obtained from the S-L method are perfectly consistent with the numerical computation in holographic $\mathrm{p}$ wave model of superfluid in GB gravity. Since in this paper we have only considered the probe limit, where the backreaction of matter fields on the metric background is neglected, it is worthy to extend the investigation to the Gauss-Bonnet holographic superfluid model away from the probe limit and take the backreaction into account. It would also be of interest to study other characteristics of these systems like the behavior of conductivity or optical features.

Acknowledgements This work was supported by the National Natural Science Foundation of China under Grant Nos. 11847092, 11775076,
11875025 and 11690034; Hunan Provincial Natural Science Foundation of China under Grant No. 2016JJ1012.

Data Availability Statement This manuscript has no associated data or the data will not be deposited. [Authors' comment: This is a theoretical study and no experimental data has been listed.]

Open Access This article is licensed under a Creative Commons Attribution 4.0 International License, which permits use, sharing, adaptation, distribution and reproduction in any medium or format, as long as you give appropriate credit to the original author(s) and the source, provide a link to the Creative Commons licence, and indicate if changes were made. The images or other third party material in this article are included in the article's Creative Commons licence, unless indicated otherwise in a credit line to the material. If material is not included in the article's Creative Commons licence and your intended use is not permitted by statutory regulation or exceeds the permitted use, you will need to obtain permission directly from the copyright holder. To view a copy of this licence, visit http://creativecomm ons.org/licenses/by/4.0/.

Funded by $\mathrm{SCOAP}^{3}$.

\section{References}

1. J. Maldacena, Adv. Theor. Math. Phys. 2, 231 (1998)

2. J. Maldacena, Int. J. Theor. Phys. 38, 1113 (1999). arXiv:hep-th/9711200

3. E. Witten, Adv. Theor. Math. Phys. 2, 253 (1998). arXiv:hep-th/9802150

4. S.S. Gubser, I.R. Klebanov, A.M. Polyakov, Phys. Lett. B 428, 105 (1998). arXiv:hep-th/9802109

5. S.A. Hartnoll, C.P. Herzog, G.T. Horowitz, Phys. Rev. Lett. 101, 031601 (2008). arXiv:0803.3295 [hep-th]

6. G.T. Horowitz, M.M. Roberts, Phys. Rev. D 78, 126008 (2008). arXiv:0810.1077 [hep-th]

7. C.M. Chen, M.F. Wu, Prog. Theor. Phys. 126, 387 (2011). arXiv:1103.5130 [hep-th]

8. H.B. Zeng, X. Gao, Y. Jiang, H.S. Zong, JHEP 1105, 002 (2011). arXiv:1012.5564 [hep-th]

9. R.G. Cai, L. Li, L.F. Li, R.Q. Yang, Sci. China-Phys. Mech. Astron. 58(6), 060401 (2015). arXiv:1502.00437 [hep-th]

10. S. Gangopadhyay, D. Roychowdhury, JHEP 08, 104 (2012). arXiv: 1207.5605 [hep-th]

11. C.Y. Lai, Q.Y. Pan, J.L. Jing, Y.J. Wang, Phys. Lett. B 749, 437 (2015). arXiv:1508.05926 [hep-th]

12. A. Akhavan, M. Alishahiha, Phys. Rev. D 83, 086003 (2011). arXiv:1011.6158 [hep-th]

13. R.G. Cai, H.F. Li, H.Q. Zhang, Phys. Rev. D 83, 126007 (2011). arXiv: 1103.5568 [hep-th]

14. Z.X. Zhao, Q.Y. Pan, J.L. Jing, Phys. Lett. B 719, 440 (2013). arXiv:1212.3062 [hep-th]

15. H.F. Li, JHEP 1107, 135 (2013). arXiv:1306.3071 [hep-th]

16. D. Roychowdhury, JHEP 05, 162 (2013). arXiv:1304.6171 [hep-th]

17. Y. Peng, Q.Y. Pan, Y.Q. Liu, Nucl. Phys. B 915 (2017). arXiv:1512.08950 [hep-th]

18. P. Basu, A. Mukherjee, H.H. Shieh, Phys. Rev. D 79, 045010 (2009). arXiv:0809.4494 [hep-th]

19. C.P. Herzog, P.K. Kovtun, D.T. Son, Phys. Rev. D 79, 066002 (2009). arXiv:0809.4870 [hep-th]

20. D. Arean, M. Bertolini, J. Evslin, T. Prochazka, JHEP 1007, 060 (2010). arXiv:1003.5661 [hep-th]

21. H.B. Zeng, W.M. Sun, H.S. Zong, Phys. Rev. D 83, 046010 (2011). arXiv: 1010.5039 [hep-th] 
22. H.B. Zeng, Phys. Rev. D 87, 046009 (2013). arXiv: 1204.5325 [hepth]

23. Y.B. Wu, J.W. Lu, W.X. Zhang, C.Y. Zhang, J.B. Zhang, F. Yu, Phys. Rev. D 90, 126006 (2014). arXiv:1410.5243 [hep-th]

24. D.G. Boulware, S. Deser, Phys. Rev. Lett. 55, 2656 (1985)

25. S.S. Gubser, Phys. Rev. D 78, 065034 (2008). arXiv:0801.2977 [hep-th]

26. R. Gregory, S. Kanno, J. Soda, JHEP 10, 010 (2009). arXiv:0907.3203 [hep-th]

27. Q.Y. Pan, B. Wang, E. Papantonopoulos, J. deOliveira, A.B. Pavan, Phys. Rev. D 81, 106007 (2010). arXiv:0912.2475 [hep-th]

28. R.G. Cai, Z.Y. Nie, H.Q. Zhang, Phys. Rev. D 82, 066007 (2010). arXiv:1007.3321 [hep-th]

29. Q.Y. Pan, J.L. Jing, B. Wang, JHEP 11, 088 (2011). arXiv:1105.6153 [gr-qc]

30. J.L. Jing, Q.Y. Pan, S.B. Chen, Phys. Lett. B 716, 385 (2012). arXiv:1209.0893 [hep-th]

31. S. Gangopadhyay, Phys. Lett. B 724, 176 (2013). arXiv:1302.1288 [hep-th]

32. W.P. Yao, J.L. Jing, JHEP 05, 101 (2013). arXiv:1306.0064 [gr-qc]

33. A. Sheykhi, H.R. Salahi, A. Montakhab, JHEP 04, 058 (2016). arXiv:1603.00075 [hep-th]

34. A. Sheykhi, A. Ghazanfari, A. Dehyadegari, Eur. Phys. J. C 78, 159 (2018). arXiv:1712.04331 [hep-th]

35. D. Parai, S. Gangopadhyay, D. Ghorai, Ann. Phys. 403, 59 (2019). arXiv:1901.10538 [hep-th]

36. C.H. Nam, Gen. Relativ. Gravit. 51, 104 (2019). arXiv:1908.06711 [hep-th]

37. M. Mohammadi, A. Sheykhi, Eur. Phys. J. C 79, 743 (2019). arXiv:1908.07992 [hep-th]

38. C.Y. Lai, Q.Y. Pan, J.L. Jing, Y.J. Wang, Phys. Lett. B 757, 65 (2016). arXiv:1601.00134 [hep-th]
39. G. Siopsis, J. Therrien, JHEP 05, 013 (2010). arXiv: 1003.4275 [hep-th]

40. G. Siopsis, J. Therrien, S. Musiri, Class. Quantum Gravity 29, 085007 (2012). arXiv:1011.2938 [hep-th]

41. H.F. Li, R.G. Cai, H.Q. Zhang, JHEP 04, 028 (2011). arXiv:1103.2833 [hep-th]

42. S. Gangopadhyay, D. Roychowdhury, JHEP 05, 156 (2012). arXiv:1204.0673 [hep-th]

43. D. Wang, M.M. Sun, Q.Y. Pan, J.L. Jing, Phys. Lett. B 785, 362 (2018)

44. M. Mohammadi, A. Sheykhi, M.K. Zangeneh, Eur. Phys. J. C 78, 984 (2018). arXiv: 1901.10540 [hep-th]

45. S.C. Liu, Q.Y. Pan, J.L. Jing, Phys. Lett. B 765, 91 (2017). arXiv: 1610.02549 [hep-th]

46. R.G. Cai, S.P. Kim, B. Wang, Phys. Rev. D 76, 024011 (2007). arXiv:0705.2469 [hep-th]

47. J. Crisostomo, R. Troncoso, J. Zanelli, Phys. Rev. D 62, 084013 (2000). arXiv:hep-th/0003271

48. M. Brigante, H. Liu, R.C. Myers, S. Shenker, S. Yaida, Phys. Rev. Lett. 100, 191601 (2008). arXiv:0802.3318 [hep-th]

49. A. Buchel, R.C. Myers, JHEP 0908, 016 (2009). arXiv:0906.2922 [hep-th]

50. G.T. Horowitz, R.C. Myers, Phys. Rev. D 59, 026005 (1998). arXiv:hep-th/9808079 [hep-th]

51. T. Nishioka, S. Ryu, T. Takayanagi, JHEP 03, 131 (2010). arXiv:0911.0962 [hep-th]

52. G.T. Horowitz, B. Way, JHEP 11, 011 (2010). arXiv:1007.3714 [hep-th]

53. M. Rogatko, I.W. Karol, JHEP 10, 152 (2016). arXiv:1608.00343 [hep-th] 